\title{
INFORME SOBRE EL RECONOCIMIENTO DEL TITULO DE LICENCIADO EN PSICOLOGÍA COMO PROFESIÓN SANITARIA
}

Aprobado en el Pleno del Consejo Asesor de Sanidad, de 22 de Diciembre de 2005

\section{RELACIÓN DE MIEMBROS DEL GRUPO DE TRABAJO}

Coordinador:

José García González.

Vocal del Consejo Asesor del Ministerio de Sanidad y Consumo.

Psiquiatra Jefe de Servicio del Hospital Universitario central de Asturias.

Amparo Belloch Fuster.

Catedrática de Psicopatología. Facultad de Psicología Universidad de Valencia.

Presidenta de la Asociación Española de Psicología Clínica y Psicopatología.

Mariano Hernández Monsalve.

Psiquiatra Jefe de Servicio. Servicios de Salud Mental de Madrid.

Expresidente de la Asociación Española de Neuropsiquiatría.

Carmen Junqué Plaja.

Catedrática de Psicología. Departamento de psiquiatría y psicobiología clínica.

Facultad de Medicina. Universidad de Barcelona

Antonio Lobo Satué.

Catedrático de Psiquiatría. Universidad de Zaragoza.

Jefe de Servicio del Hospital Clínico Universitario de Zaragoza.

Vicente Pelechano Barberá.

Catedrático de Personalided, Evaluación y

Tratamientos Psicológicos.

Universidad de La Laguna. Tenerife

\section{INDICE}

1. Origen, delimitación del problema y estado de la cuestión. 222

1.1. La creación de los estudios de Psicología en España y datos de la situación actual

1.2. Normativas sanitarias que afectan a los profesionales de psicología .. 223

1.3. El conflicto actual y problemas .. 225

1.4. Posiciones discrepantes ............ 225

2. Análisis de la formación básica y curricular de la Licenciatura de Psicología

2.1. Líneas maestras del plan de estudios vigente

3. Normativa comparada con otros países de la U.E. en la formación de la licenciatura y acceso al ejercicio profesional

3.1. Requisitos generales de formación

3.2. Acceso al ejercicio profesional independiente

3.3. El futuro: el diploma europeo en psicología

3.4. Conclusiones de la revisión de normativas europeas

4. Sobre la regulación y formación de las profesiones sanitarias

5. Diferencias entre el ejercicio de profesiones sanitarias y la actividad de otras profesiones en el ámbito sanitario ... 234

6. La creación de la especialidad de psicología clínica y la regulación de su sistema de formación como garantía de un alto nivel profesional

7. Sobre la necesidad y demanda de psicólogos clínicos en el Sistema Nacional de Salud

8. Síntesis y conclusiones

9. Recomendaciones 239

Anexo I.

Regulación de los estudios de psicología en diversos paises Europeos

Documentación y bibliografía 


\section{ORIGEN, DELIMITACIÓN DEL PROBLEMA Y ESTADO DE LA CUESTIÓN}

\subsection{La creación de los estudios de Psicología en España y datos de la situación actual.}

La creación de una Licenciatura independiente de Psicología no tuvo lugar hasta el año 1975, aunque la presencia de disciplinas psicológicas en el ámbito universitario español se produce de manera regular en los años 50, evolucionando progresivamente.

En los primeros años conviven las materias propiamente psicológicas con las de otras Licenciaturas con las que comparten titulación.

A partir de 1980 , se puede hablar con propiedad de que los estudios de la Licenciatura en Psicología en España incluyen únicamente contenidos exclusivos y / o propios de Psicología, los cuales van siendo paulatinamente ofertados por un buen número de Universidades, hasta llegar a un total de 31 (29 presenciales, 2 a Distancia) en el curso 200304. Esta cifra sitúa la Licenciatura de Psicología en el tercer lugar en cuanto a número de estudiantes matriculados en todo el Estado.

Según los datos manejados por la Conferencia de Decanos de Facultades de Psicología -Libro Blanco sobre la Titulación-, durante 4 cursos académicos, desde 1999-00 hasta 2002-03, el número total de estudiantes que obtuvo el grado de Licenciado en Psicología en 22 Universidades públicas fue de 19.432 , y de 2.069 en las 8 Universidades privadas que imparten la Licenciatura: por tanto, en un período de 4 años, han egresado un total de 21.501 psicólogos de la Universidad Española.

En 1990 se publica el RD 1428/1990, de 20 de Noviembre, que establece las directrices generales por las que deben regirse los planes de estudio conducentes al título universitario de Licenciado en Psicología, y que, con algunas modificaciones, continua vigente. En dicho $\mathrm{RD}$ se afirma que las enseñanzas deberán proporcionar una formación científica adecuada en los aspectos básicos y aplicados de la Psicología (artículo único). Este objetivo, $\tan$ genéricamente expresado, resalta úni- camente el carácter científico de la formación y el hecho que deberá atenderse tanto a los contenidos básicos como a los aplicados.

Si comparamos esta formulación con las que aparecen en los decretos de las licenciaturas sanitarias, que definen de manera expresa sus contenidos y objetivos ligados expresamente a la formación para la intervención en la salud, podemos valorar que la Licenciatura de Psicología ha sido considerada desde su creación como una formación generalista y polivalente, que como cualquier otra Licenciatura en España habilita para el ejercicio profesional de la psicología en una relativamente amplia variedad de actividades y contextos, que van desde el educativo y escolar, al laboral, empresarial, militar, judicial, penitenciario, y al sanitario. Dado el carácter generalista de la licenciatura es lógico suponer, por tanto, que para el mejor y más capacitado desempeño profesional específico en alguna de estas actividades y contextos, será necesaria una especialización post-graduada, si bien el título de licenciado habilita para la intervención en los diversos campos de la Psicología.

Efectivamente, las actividades del licenciado en psicología, de manera acorde con la concepción generalista que subyace en los estudios de licenciatura, se vienen realizando en campos muy diversos. Así se reconoce en una publicación del Colegio Oficial de Psicólogos, de 1998, el documento titulado «Perfiles Profesionales del Psicólogo" ${ }^{1}$, en el que se establece un total de 8 perfiles profesionales diferenciados:

- Psicología de la Actividad Física y el Deporte

- Psicología Clínica y de la Salud

- Psicología de las Drogodependencias

- Psicología de la Educación

- Psicología de la Intervención Social

- Psicología Jurídica

- Psicología del Trabajo y de las Organizaciones

- Psicología del Tráfico y de la Seguridad

En este documento se delimitan las competencias y capacidades específicas para los psicólogos en cada uno de estos ámbitos de

${ }^{1}$ Disponible en www.cop.es/perfiles/index.html 
actuación profesional. Consecuentemente, se plantean requisitos y necesidades de formación específica y diferencial para cada uno de tales perfiles, incidiendo en el hecho de que esa formación debe ser adquirida después de la consecución del grado de Licenciado en Psicología, que a su vez, se considera polivalente y generalista.

Otro aspecto al que hay que hacer referencia es el bagaje curricular de los alumnos que pueden acceder a los estudios de Psicología, pues éste determina en buena medida el nivel en que algunos contenidos pueden ser explicados y evaluados. El actual sistema de acceso a la Universidad permite la entrada a los estudios de Psicología a alumnos de cualquier opción de bachillerato. La opción de Ciencias de la Salud es claramente minoritaria entre los alumnos que acceden a los estudios de Psicología. Nuevamente, aparece clara la opción del legislador de considerar la titulación de Psicología como una formación de características muy amplias y poco específicas.

\subsection{Normativas sanitarias recientes que afectan a los profesionales de la psicología}

La actividad de los profesionales de la psicología en el ámbito sanitario se mantuvo básicamente sin ninguna regulación normativa que estableciera su diferenciacion hasta la creación de la especialidad de psicología clínica por el R.D 2490/ de 1998

Desde 1998, y más precisamente en los últimos años, se han producido una serie de normativas que han venido a ordenar y regular la autorización centros y establecimientos sanitarios y las profesiones sanitarias..

Mediante el Real Decreto 2490/98, que crea la especialidad sanitaria y el título de especialista en psicología clínica, se establecen los requerimientos para la obtención de esta especialidad y, al tiempo, el sistema de formación - Modelo de Interno Residente, semejante al del resto de las especialidades sanitarias, el acceso a la misma, su organización y evaluación, la acreditación de los centros y unidades sanitarios que ejercerán los cometidos docentes, y la creación de la Comisión Nacional de la Especialidad de
Psicología Clínica. Se determinan también funciones y competencias para quienes tienen esa titulación y dedica cuatro Disposiciones Transitorias a agrupar y establecer los requisitos para acceder a la homologación del título a los profesionales y académicos en ejercicio.

Así mismo, se señala, en la Disposición Adicional Segunda aplicable al Personal Estatutario, que este nuevo especialista cuando preste servicios en las Instituciones sanitarias públicas «estará incluido en el ámbito de aplicación del Estatuto Jurídico de Personal Médico de la Seguridad Social al que accederá por el procedimiento establecido para los facultativos especialistas».

Este RD 2490/1998 se ha desarrollado mediante la orden PRE/1107/ 2002 en la que se establecen las vías transitorias de acceso al título y se ha ampliado recientemente con la publicación del Real Decreto 654 /2005, de 6 de Junio, por el que se modifican las disposiciones transitorias del anterior ampliando los plazos para la solicitud de homologación del título de especialista a licenciados y académicos en ejercicio.

Posteriormente, en 2003, se publica, el RD $1277 / 03$, que establece las bases generales sobre autorización de centros, servicios y establecimientos sanitarios. Este RD desarrolla la Ley de Cohesión y Calidad del SNS y pretende fijar criterios y parámetros homogéneos en el Estado para el reconocimiento del carácter sanitario de actividades profesionales y centros. Corresponde a las Consejerías de Salud de cada CA registrar como sanitarios los centros, servicios y establecimientos que así lo soliciten y cumplan con aquellos criterios.

Pero este R.D., que tiene un carácter general y no es específico para la Psicología, tiene deficiencias en la definición de unidades y servicios y ha dado lugar a problemas con su aplicación. Todo ello, sin perjuicio, de que el propio decreto establece que «... no es propósito de esa norma ordenar las profesiones sanitarias, ni limitar las actividades de los profesionales, sino sentar las bases para las garantías de seguridad y calidad de la atención sanitaria».

La Comisión Nacional de la especialidad ha propuesto ya en su momento su modifica- 
ción. Así mismo, por razones similares el COP (Colegio Oficial de Psicólogos) ha denunciado sus deficiencias y exigido cambios.

Además de las insuficiencias señaladas por el COP, presenta otras inadecuaciones y, en algunos casos, contradicciones con el estatus real del desempeño profesional de los psicólogos en el SNS, que han sido señaladas también por diversas asociaciones científicas y profesionales de la Psicologia (AEN, AEPCP, ANPIR, ), que han reclamado modificaciones del $R D .1277 / 2003$ o su retirada hasta tanto no se introduzcan las correcciones pertinentes a la psicología y los psicólogos.

Lo cierto es que hay un acuerdo general entre diversas asociaciones científicas y profesionales acerca de que este decreto es inadecuado en la definición que hace de centros, unidades asistenciales y establecimientos sanitarios, y en que utiliza concepciones ya superadas, tanto desde el punto de vista científico como profesional y asistencial.

Finalmente, se promulga la ley 44/2003 de ordenación de las profesiones sanitarias LOPS-, en la que se definen aquéllas licenciaturas y especialidades que por su formación y campo de intervención son inequívoca y plenamente sanitarias, para proceder a su regulación y en ella solamente se incluye como profesión sanitaria a los psicólogos que estén en posesión del título de especialista en psicología clínica. $^{2}$

La LOPS no entra en la limitación del ejercicio profesional a los licenciados de ninguno de los títulos oficiales existentes a los que habilita el $\mathrm{M}^{\mathbf{2}}$ de Educación, a través de la titulación oficial. Sí aborda, en cambio, aspectos muy relevantes para el establecimiento y regulación de definiciones, criterios, procesos, órganos, formación, carrera profesional y otros como garantía de calidad de las profesiones sanitarias.

Así lo establece el texto de la Ley cuando en su Exposición de motivos dice que .... esta

\footnotetext{
${ }^{2}$ La LOPS incluye como profesiones sanitarias del nivel de licenciado: licenciados en medicina, farmacia, odontología, veterinaria y quienes se encuentren en posesión de un título oficial de especialista en Ciencias de la Salud establecido, conforme a lo previsto en su artículo 19.1, para psicólogos, químicos, biólogos y bioquímicos.
}

ley tiene por finalidad dotar al sistema sanitario de un marco legal que contemple los diferentes instrumentos y recursos que hagan posible la mayor integración de los profesionales en el servicio sanitario, en lo preventivo y en lo asistencial, tanto en su vertiente pública como en la privada, facilitando la corresponsabilidad en el logro de los fines comunes y en la mejora de la calidad de la atención sanitaria prestada a la población, garantizando, asimismo, que todos los profesionales sanitarios cumplen con los niveles de competencia necesarios para tratar de seguir salvaguardando en derecho a la protección de la salud»

\subsection{El Conflicto y problemas actuales}

La situación de conflicto y debate presentes entre la comunidad científico-profesional de los psicólogos en la actualidad se inicia con la publicación de las dos últimas iniciativas legislativas mencionadas en el punto anterior. Su publicación y aplicación han dado lugar a discrepancias y ostensibles protestas de un amplio sector de psicólogos, principalmente los Colegios de Psicólogos, la Conferencia de Decanos de las Facultades de Psicología y estudiantes, que reclaman que la licenciatura de psicología sea reconocida e incluida en la LOPS como una profesión sanitaria, exigiendo que a esos efectos se modifique la ley.

Esas protestas colisionan con las posiciones de otros sectores vinculados con la Psicología clínica - AEN, AEPCP, MGPC, ANPIR ${ }^{3}-\mathrm{y}$ dos Colegios profesionales: el Colegio Oficial de Psicólogos de Andalucía Occidental y el Colegio Oficial de Psicólogos de Galicia, que han manifestado documentalmente no suscribir el criterio de las entidades anteriores.

\subsection{Posiciones discrepantes}

1.4.1. Los argumentos en los que fundamentan la defensa de la licenciatura como

\footnotetext{
${ }^{3}$ Asociación Española de Neuropsiquiatría, AEN; Asociación Española de Psicología Clínica y Psicopatología, AEPCP; Mesa Galega de Psicología Clínica 0,MGPC; Asociación Nacional de Psicólogos Internos y Residentes, ANPIR.
} 
profesión sanitaria los Colegios de Psicólogos y la Conferencia de Decanos se resumen en los siguientes:

- La licenciatura de psicología, aunque no está adscrita en el $\mathrm{M}^{\mathrm{a}}$ de Educación al área de ciencias de la salud, tiene en su curriculum muchas materias de contenido y naturaleza sanitaria y otras relacionadas con la salud y, en consecuencia, forma y prepara a estos licenciados para ejercer en el campo de la salud.

- El licenciado en psicología realiza fundamentalmente actividades sanitarias en los múltiples ámbitos de su actuación y, por tanto, estos profesionales han de ser considerados como profesionales sanitarios.

- Si no se reconoce el título en psicología como profesión sanitaria, no se podrá disponer de la oferta necesaria de psicólogos para el sector sanitario, porque el número de plazas para la formación de especialistas en psicología clínica es muy reducido

- No considerar el título de licenciado en psicología como una profesión sanitaria expresa una concepción desfasada y muy restringida de la salud. Es incuestionable que el comportamiento humano tiene una influencia decisiva sobre la salud física y psicológica de los individuos y la psicología es la ciencia que estudia el comportamiento hu$\operatorname{mano}^{4}$

1.4.2. Posiciones y argumentos de otras entidades y asociaciones ${ }^{5}$ que no comparten el criterio de considerar el título licenciado en psicología como profesión sanitaria, ni que su actividad sea sanitaria en su totalidad.

- La formación de la licenciatura en psicología no se dirige especifica y fundamentalmente a dotar a los interesados

\footnotetext{
${ }^{4}$ Resumen del informe de los COP contestando al enviado por el Ministerio de Sanidad al defensor del Pueblo. Infocop Suplemento informativo de Papeles del Psicólogo, No 22,2005.

${ }^{5}$ Las mencionadas en la Nota 2.
}

de los conocimientos, habilidades y actitudes propias de la atención a la salud: Un porcentaje de un $18 \%$ a un $25 \%$ de las materias impartidas pertenecen al ámbito de la salud y habilita a los licenciados a desarrollar actividades sanitarias, pero la globalidad de la formación es polivalente. Consiguientemente, no se ajusta a lo que la LOPS define como Profesiones sanitarias tituladas y reguladas en su Artíc.2: «De conformidad con el artículo 36 de la Constitución, y a los efectos de esta Ley, son profesiones sanitarias, tituladas y reguladas, aquellas cuya formación pregraduada o especializada se dirige específica y fundamentalmente a dotar a los interesados de los conocimientos, habilidades y actitudes propias de la atención a la salud..... Y, precisamente, al no estar estos estudios de la licenciatura dirigidos específica y fundamentalmente a la salud, no resulta de aplicación el Artic. 13 de la LOPS que, entre otros asuntos relevantes establece que " la determinación del número de alumnos admitidos a la formación pregraduada responderá a las necesidades de profesionales sanitarios y a la capacidad existente para su formación".

- El perfil profesional vigente de los licenciados en psicología es el de un psicólogo generalista y polivalente: esto quiere decir, que los recién licenciados pueden orientar su actividad hacia diversos campos posibles para los que tienen los suficientes conocimientos básicos. Estos ámbitos hacen referencia a aspectos tan diversos como los recursos humanos, la selección de personal, los estudios de mercado y opinión de los consumidores, la ergonomía, la mejora del rendimiento escolar y del aprendizaje, la detección de necesidades educativas especiales, la optimización del rendimiento deportivo, la psicología militar, la seguridad vial, además de por supuesto la psicología clínica y de la salud. El propio Colegio ${ }^{6}$

\footnotetext{
${ }^{6}$ Véase el documento sobre Perfiles Profesionales de los Psicólogos, publicado en 1998 y disponible en www.cop.es/perfiles/index.html
} 
Oficial de Psicólogos ha confirmado esta tesis en un amplio documento, ya citado.

Para obtener la capacitación apropiada y el desempeño cualificado de todos esos perfiles de actividad existe formación post-licenciatura, tal y como se establece en el ámbito universitario -de ahí la proliferación de masters y cursos de postgrado relativos a todos esos ámbitos- y por la organización colegial (Colegio Oficial de Psicólogos).

- A través del proceso de homologación del título de especialista en Psicología Clínica, y mediante el aumento de plazas para la formación por el sistema PIR es previsible una disponibilidad adecuada para satisfacer las demandas de psicólogos clínicos en el sistema sanitario. Se han presentado más de 10.000 solicitudes de homologación del título que están en proceso de estudio por la Comisión Nacional de la Especialidad, y se incrementaran con el nuevo R.D 654 / 2005.

- Las discrepancias existentes entre diversos sectores de la Psicología no tienen su origen en la LOPS, sino en concepciones e intereses distintos sobre los estudios de licenciatura, la formación y ejercicio profesional de los especialistas en Psicología clínica y los procedimientos necesarios para garantizar el derecho a la salud que tienen los ciudadanos

\section{ANÁLISIS DE LA FORMACIÓN BÁSICA Y CURRICULAR DE LA LICENCIATURA DE PSICOLOGÍA}

La falta de concreción de objetivos sanitarios en los decretos de la titulación de la licenciatura de Psicología contrasta con los reales decretos que establecen los planes de estudios de titulaciones sanitarias como Farmacia, Medicina, o Veterinaria, en las que los objetivos se describen de forma pormenorizada y en ellos se hacen numerosas menciones a los aspectos clínicos y de la salud que comporta la formación.

De hecho, en el articulado de los reales decretos que establecen los planes de estu- dio no se menciona el carácter sanitario de la carrera de Psicología. En este sentido, el articulado del título de Licenciado no se asemeja al de los otros títulos de naturaleza sanitaria y en cambio es idéntico a otros títulos con objetivos formativos similares. Es particularmente ilustrativo el caso del título de Licenciado en Biología (Real Decreto 387/1991) en el que se define el objetivo de la titulación con las mismas palabras que en el de Psicología, substituyendo ésta por Biología. En ambos casos los respectivos planes de estudio ponen de manifiesto que la formación no va dirigida primordialmente a la formación de profesionales de la salud, si bien, también en ambos casos es posible el acceso a la profesión sanitaria a través de la formación especializada en ciencias de la salud.

Si nos atenemos a la organización de las materias troncales que establece el mencionado RD 1428/1990, de 20 de Noviembre, observamos que de los 300 créditos que como mínimo debe suponer la carga lectiva, se fijan únicamente los contenidos obligatorios de 137 créditos (aproximadamente el 45 $\%)$. Estos valores contrastan con los de la titulación de Medicina en la que se fijan 400 (80\%) de los 500 créditos mínimos, o la de Farmacia en la que se fijan 193 (aproximadamente el $64 \%$ ) de los 300 créditos mínimos de la titulación. Es fácil entender que una titulación que deja a la libre disposición de las universidades más de la mitad de sus contenidos ha de ser considerada necesariamente poco específica. No parece, pues que el legislador haya considerado necesario fijar un modelo de formación que capacite para un ámbito de aplicación definido y mucho menos que presuponga una formación en el campo de las ciencias de la salud, cualquiera que sea la definición que hagamos de ésta.

El estudio de los contenidos mínimos de troncalidad obligatoria que fijan las directrices generales pone de manifiesto que no se hace mención expresa de las palabras salud, sanidad y clínica que caracterizan los contenidos de las titulaciones propias de las Ciencias de la Salud. Existen algunos pocos contenidos que también aparecen en otras titulaciones sanitarias como: principios de 
genética y evolución, fundamentos de neurociencia, estadística descriptiva, estadística inferencial, etc., pero que por su naturaleza también son propias de otras titulaciones no sanitarias, por lo que no pueden ser considerados contenidos específicos de la formación sanitaria. Otros contenidos tales como, aprendizaje escolar, tipología de las organizaciones, mecanismos de razonamiento y solución de problemas, no son característicos de las titulaciones sanitarias. Los contenidos más específicos los encontramos en las materias Evaluación psicológica y Psicopatología y Técnicas de intervención y tratamiento psicológico que, juntamente con los contenidos que la titulación comparte con las titulaciones sanitarias, pueden constituir un punto de partida para la formación sanitaria posterior, pero que por sí solas resultan claramente insuficientes.

Una materia troncal con unas características especiales es el Practicum, un tipo de prácticas tuteladas semejante al de algunas titulaciones sanitarias, pero que también existe en otras titulaciones con características semejantes aunque más estrictas, como por ejemplo, en la licenciatura de Derecho. La dificultad para homologar esta materia con las equivalentes en las titulaciones sanitarias (por ejemplo, Farmacia o Veterinaria) es que pueden tener cualquier contenido relacionado con alguno de los ocho perfiles profesionales ya descritos y mencionados con antelación y no tener relación alguna con la actividad sanitaria, aunque en otros casos se realicen en este ámbito (hospitales, servicios de salud, etc.).

\subsection{Líneas maestras del plan de estudios}

En síntesis, las líneas maestras del plan de estudios, vigente en la actualidad, se articulan en seis grandes áreas:

a) Contenidos especificos propios de la psicología, agrupados en torno al área de conocimientos de "Psicología Básica», que incluye disciplinas como: Historia de la Psicología, Atención, Percepción, Memoria, Aprendizaje, Pensamiento, o Lenguaje, (los denominados procesos psicológicos bási- cos). Tales contenidos configuran los fundamentos de la Psicología, sus señas de identidad como disciplina científica. En consecuencia, ocupan la mayor parte de la carga lectiva que deben superar los estudiantes: $24,08 \%$ de la troncalidad, independientemente de cuál sea su futuro ámbito de desempeño profesional.

b) Contenidos técnico-metodológicos, agrupados en el área de Metodología y Estadística: incluyen materias como estadística, construcción de instrumentación psicológica, análisis de datos, métodos y diseños de investigación. Es decir, contenidos característicos y necesarios de cualquier ámbito científico. En términos porcentuales, el peso de estas materias en la troncalidad de la Licenciatura es del $11,67 \%$.

c) Fundamentos biológicos del comportamiento humano: Estos contenidos se agrupan en el área de Psicobiología y su finalidad es proporcionar a todos los psicólogos los rudimentos de los aspectos biológicos que subyacen al comportamiento humano. Su peso porcentual en la troncalidad de los estudios es escaso $(11,67 \%)$, e incluye contenidos como genética del comportamiento humano y animal, etología, psicofisiología, psiconeurología, etc.

d) Fundamentos sociales del comportamiento humano: Se agrupan en el área de Psicología Social y de las organizaciones, y representa el $13,13 \%$ de la troncalidad. Sus contenidos disciplinares inciden en el conocimiento de las personas como individuos sociales, y por tanto se estudian disciplinas como dinámica comportamental de los grupos y las organizaciones, psicología laboral y del trabajo, relaciones individuo-sociedad, etc. Proporciona la formación básica para una posterior especialización del psicólogo en uno de los ámbitos tradicionales de trabajo de la Psicología: Psicología del trabajo y de las organizaciones, principalmente, si bien se está progresivamente abriendo a otros campos de intervención.

e) Fundamentos evolutivos y de desarrollo del comportamiento humano: Estos contenidos tienen como finalidad la introducción a los aspectos vinculados con la psicología evolutiva y del desarrollo, con una fuerte implicación de los mismos en el contexto educati- 
vo. Su representación en el plan de estudios vigente es del 14,59\%. El objetivo principal es introducir al estudiante en el segundo de los ámbitos tradicionales de la profesión: la psicología de la educación, así denominada en el documento sobre perfiles profesionales del psicólogo al que antes se hizo mención, en el que se recuerda que la consolidación del psicólogo educativo como profesional con competencias propias, se produce sobre todo con la publicación de la LOGSE, y en esa consolidación "han confluido tres grandes líneas de actuación: (...) un enfoque sociolaboral, que pretendía la inserción eficaz y madura del alumno en la vida activa (funciones de orientación profesional, asesoramiento vocacional), Otra línea de actuación es de tipo psicológico y pedagógico y pretende la mejora de la vida escolar, en una línea de apoyo a la comunidad educativa (funciones de asesoramiento, de formación de apoyo didáctico...). Una tercera (...) de orientación terapéutica (reeducadora, compensadora...)", siendo el contexto escolar el ámbito en el que se llevan a cabo tales funciones (e.g., gabinetes de orientación psicopedagógica).

f) Fundamentos psicológicos de las alteraciones, trastornos y enfermedades mentales y del comportamiento: Agrupa disciplinas características de la psicología clínica, que es el tercer ámbito de desarrollo profesional tradicional de los psicólogos: psicología de la personalidad, diferencias humanas, psicopatología, psicoterapias (en sus diferentes modalidades), evaluación y diagnóstico, que en conjunto tienen un peso porcentual total del $18,24 \%$.

Las tres últimas áreas refieren, como se ha dicho, a los tres ámbitos profesionales tradicionales de la psicología: trabajo, educativo, y clínico. En consecuencia, algunas Facultades (Complutense de Madrid, Autónoma de Barcelona) han diseñado una intensificación curricular de cada una de ellas, lo que se traduce en «itinerarios preprofesionales" que se cursan en el segundo ciclo y que ocupan la mayor parte del peso formativo. Según los datos aportados en el documento de la Conferencia de Decanos mencionado más arriba, en las Facultades en que existen tales itinerarios, el 40-50\% de los estudiantes escogen el de Clínica, el 20-25\% el de Social/organizacional, y solo el $5-10 \%$ el de Educativa (datos de la UCM y la UAB).

En la Tabla 1 se resumen estos datos (obtenidos del informe de la reunión de los Decanos de Psicología celebrada en Murcia el pasado mes de mayo).

Tabla 1. Materias troncales y números de créditos asignados en los planes de estudio vigentes

\begin{tabular}{llcc}
\hline Área de conocimiento & Disciplinas troncales & Créditos $\begin{array}{c}\text { \% sobre } \\
\text { el total }\end{array}$ \\
\hline 1. Básica & $\begin{array}{l}\text { Historia. Procesos psicologicos básicos (aten- } \\
\text { ción, pensamiento, memoria, lenguaje, etc..) }\end{array}$ & 33 & $\mathbf{2 4 , 0 8 \%}$ \\
\hline 2. Metodología & $\begin{array}{l}\text { Análisis de datos, métodos técnicas y diseños } \\
\text { de investigación, estadística, etc. }\end{array}$ & 16 & $11,67 \%$ \\
\hline 3. Psicobiología & $\begin{array}{l}\text { Bases biológicas de la conducta, genética, psi- } \\
\text { coendocrinologia, etc. }\end{array}$ & 16 & $11,67 \%$ \\
\hline 4. Social & Ps. Social, Organizaciones, Grupos & 18 & $13,13 \%$ \\
\hline 5. Evolutiva y de la Educación & $\begin{array}{l}\text { Educación e Instrucción, Ps. Escolar, ciclo vital, } \\
\text { etc.. }\end{array}$ & 20 & $14,59 \%$ \\
\hline 6. Clínica & $\begin{array}{l}\text { Evaluación, Diagnóstico, Personalidad, Diversi- } \\
\text { ded, Tratamientos, Psicopatología }\end{array}$ & 25 & $18,24 \%$ \\
\hline Prócticum & & 9 & $6,56 \%$ \\
\hline TOTAL CRÉDITOS TRONCALES & 137 & & \\
\hline
\end{tabular}


3. NORMATIVA COMPARADA CON OTROS PAISES DE LA U.E. EN LA FORMACIÓN DE LA LICENCIATURA Y ACCESO AL EJERCICIO PROFESIONAL

En este punto se revisan los requisitos vigentes para la cualificación profesional de los psicólogos en Europa, tomando como referencia la documentación al respecto que se encuentra disponible en www.europsych.org.

\subsection{Requisitos generales de formación para la obtención de la licenciatura en psicología: planes de estudio vigentes}

Todos los países europeos están actualmente en proceso de reestructuración de sus planes de estudio con el fin de adecuarlos al Espacio Europeo común de educación superior. Por tanto, en la actualidad conviven los modelos antiguos con la implantación paulatina del nuevo esquema, que es el que se contempla en los recientes RD 55/2005 y 56/2005 (BOE 21 Enero) sobre los estudios de grado y postgrado.

De todos modos, en la mayoría de los países europeos los estudios equivalentes a los de la Licenciatura de Psicología de España, se han venido organizando tradicionalmente siguiendo ya ese esquema que establecen los decretos mencionados anteriormente, con las lógicas particularidades de cada Estado y, dentro de cada uno de ellos, de cada región, comunidad, o nacionalidad. En todos los casos, los estudios de grado no capacitan para el ejercicio profesional (su duración está entre 2 y 4 años), pero sí lo hacen los de postgrado (de entre 2 y 4 años, según la especialidad profesional escogida). En el anexo I se ofrece un resumen de los planes de estudio de algunos de los países europeos, con el fin de poder realizar una adecuada comparación con los vigentes en España (ver Anexo I)

\subsection{Acceso al ejercicio profesional independiente}

En la mayoría de estos Países se requieren estudios posteriores a la Licenciatura (que, en general es de 5 años) para ejercer como profe- sional_independiente en alguna de las áreas tradicionales de la Psicología. La razón estriba en que, aunque la licenciatura teóricamente capacita para el ejercicio profesional, al no existir una profesión de "psicólogo generalista» por así llamarla, sino psicólogos especialistas en algún campo concreto (educativo, clínico, de la salud, neuropsicologia, trabajo y organizaciones, etc..) es imprescindible realizar formación adicional posterior a los 5 años de licenciatura para el ejercicio profesional independiente en alguno de los campos señalados.

En el caso específico de la Psicología Clínica, los requisitos para el ejercicio profesional son los más exigentes con diferencia, y en términos generales se requiere un entrenamiento adicional posterior de, al menos, 3 años.

No se contempla la posibilidad de que un psicólogo pueda ejercer como tal en el ámbito sanitario (sea cual sea la especialidad sanitaria: clínica, salud, psicoterapia, psiconeurología, etc.), sin haber realizado especialización post-licenciatura de, al menos, 3 años, en los que necesariamente debe haber un peso específico importante de prácticas regladas y supervisadas.

\subsection{El futuro: el diploma europeo en psicología}

La mayoría de los países están adaptando sus curricula a las directrices generales aprobadas por la European Federation of Psychologists Associations (EFPA) (en la cual han participado activamente, entre otras, la Conferencia de Decanos española, la Junta estatal del Colegio Oficial de Psicólogos, o el Colectivo de Representantes de Estudiantes de Psicología de las Facultades Españolas).

La estructura del Diploma Europeo, se ajusta en líneas generales a los RD 55/2005 y 56/2005 (BOE 21 Enero) sobre los estudios de grado y postgrado, mencionados al inicio, aunque comporta un mayor nivel de exigencia que el que se desprende de tales decretos.

Se estructura en tres fases, con una duración mínima de 6 años (360 Créditos Europeos, $\mathrm{CE}$ e a delante; $1 \mathrm{CE}=25$ horas).

- Bachelor o equivalente: Formación básica generalista en psicología. No proporciona cualificación profesional, y por 
tanto no capacita para el ejercicio profesional independiente. Duración $180 \mathrm{CE}$ (3 años aprox.)

- Master o equivalente: Preparación para la práctica profesional independiente. Duración: $120 \mathrm{CE}$ ( 2 años). El año $1^{\circ}$ está ocupado con contenidos teóricos; el año $2^{\circ}$ se reparte por igual para la realización de una tesis (investigación aplicada) y práctica pre-profesional supervisada. Sus contenidos pueden ser:

a) Indiferenciados (i.e., no relacionados con un área profesional en particular). Sirven para el acceso al grado de Doctor (de contenidos claramente investigadores) o para la práctica profesional como psicólogo generalista, no especializado.

b) Diferenciados: preparan para la práctica profesional en algún ámbito concreto de estos tres: clínica, trabajo/organizaciones, educativa.

El documento explicita: "Debe quedar claro que el Master o grados equivalentes proporcionan la cualificación básica necesaria para introducirse en la práctica de la psicología, y es necesario que se continúe con un periodo de práctica supervisada antes de que un individuo pueda ser considerado competente como profesional independiente. La práctica profesional especializada en cualquier área de la psicología requerirá en general un entrenamiento postacadémico en áreas tales como psicología de la salud, psicología clínica, psicología del trabajo y las organizaciones, psicología educativa, psicología infantil, etc.." (pág. 22).

- Práctica formativa supervisada: Su objetivo es iniciar al estudiante a la práctica profesional en un área concreta de la psicología. Duración: 1 año (60 CE). El tipo de trabajo se concibe como semi-independiente, es decir, bajo supervisión de otro psicólogo en un contexto profesional acreditado oficialmente para tal fin y en un centro acreditado.

Una vez superadas las tres fases, el estudiante estará en condiciones de optar al título de Diploma Europeo en Psicología, análogo al actual de Licenciado en Psicología, que se concibe como un título generalista que capacita para la práctica profesional independiente de la psicología, sin el rango de especialista. Para esto último será necesario realizar una formación específica posterior, según las normativas de cada País. El documento establece tres áreas de especialización profesional: Educativa, Salud, y Trabajo, y una cuarta (Otras) en la que se incluirían las particularidades de cada Estado (por ejemplo, y en la línea de lo que se examinó en el primer punto, neuropsicología, psicología del desarrollo, psicología política, etc..).

\subsection{Conclusiones de la revisión de normativas europeas}

La definición de la Psicología como «disciplina que se ocupa de los procesos mentales y sus manifestaciones comportamentales» 0 , que se recoge en el documento «A European framework for psychologists' training» (EuroPsy'T), financiado por el programa Leonardo da Vinci, no hace mención a la especificidad sanitaria de la Psicología. Antes bien, resalta su carácter multiforme, al afirmar que "es una ciencia que trata todos y cada uno de los aspectos en los que se manifiesta el comportamiento de las personas, incluyendo su interacción con el entorno, la invención de nuevas ideas, y la interacción social. Trata de las cuestiones generales sobre las causas de las diversas actividades humanas, así como de los problemas de los individuos, los grupos, y los sistemas sociales. Asimismo es relevante para entender diversos ámbitos de la vida cotidiana, como la escuela, el trabajo, el ocio, la actividad deportiva, la creatividad y el desarrollo de uno mismo. Es decir, la Psicología trabaja sobre la rica variedad de procesos de pensamiento, de personalidad, y de actividad que caracterizan el funcionamiento humano y el desarrollo en sociedad, incluyendo el comportamiento problemático». En definitiva, los aspectos sanitarios (referidos en esta definición como comportamientos problemáticos) son una parte de la psicología, y no el todo

La duración media de los estudios vigentes para la obtención del equivalente español al grado de Licenciatura en Psicología, es de cinco años, estructurados en dos ciclos. 
El segundo de esos ciclos - que lleva aparejado el título de Master en...- suele capacitar para el ejercicio profesional en algún ámbito profesional específico, excepto para el caso de la Psicología Clínica y sus variantes, que habitualmente requieren estudios adicionales, con una duración de 3 años o más.

En estos Países las asociaciones científicas y profesionales establecen explícitamente la necesidad de realizar estudios post-licenciatura específicos para trabajar como psicólogo en contextos sanitarios. Para ello se requieren especialidades como psicología clínica, psicoterapia, psicología de la salud, o neuropsicología, entre otras. Estos estudios tienen una duración media de 3 años, e incluyen de manera prioritaria formación práctica bajo supervisión. El acceso a ellos es restringido y requiere en general calificaciones previas altas.

Las nuevas directrices para el Diploma Europeo de Psicología (que sería el análogo a la Licenciatura actual), reproducen el esquema que se acaba de resumir, pero además lo amplía: son necesarios 6 años para optar a Diploma (o Licenciatura generalista), siendo el último de práctica supervisada. Una vez obtenido el Diploma, que capacita para el ejercicio profesional independiente como psicólogo generalista o no especialista, se requieren cursos posteriores de especialización para los tres campos profesionales tradicionales de la psicología: Salud-Clínica, Educativa-Escolar, Trabajo-Organizaciones.

No se contempla la figura del psicólogo generalista como capaz de asumir tareas profesionales sin supervisión en el ámbito sanitario.

Los contenidos curriculares de las distintas especialidades profesionales post-licenciatura (Educativa, Trabajo, Salud), son muy distintos entre sí. Únicamente el de salud recoge contenidos referidos a la aplicación de los conocimientos psicológicos en el ámbito sanitario.

\section{SOBRE LA REGULACIÓN Y FORMACIÓN DE LAS PROFESIONES SANITARIAS}

Las actividades en el campo de la salud se han diversificado y hecho más complejas. El ámbito de conocimientos y habilidades de las profesiones sanitarias, incluyendo los propios de las especialidades, es ya demasiado amplio y diverso como para que pueda ser abarcado por una única profesión. Se hace imprescindible la concurrencia de otras disciplinas y profesiones que conformen el campo interdisciplinario de las ciencias de la salud. Gestores, economistas de la salud, biólogos, sociólogos, entre otros, serán profesiones que concurrirán cada vez más en el campo de las actividades sanitarias conformando una multi-disciplinariedad con la interacción y el desarrollo de actividades de profesiones distintas.

La LOPS ha venido a establecer una regulación de las profesiones sanitarias tituladas, en lo referente al ejercicio profesional, a la estructura general de la formación y a su desarrollo profesional. Y define, como antes quedó señalado y a esos efectos,» como profesiones sanitarias tituladas y reguladas aquellas cuya formación pregraduada o especializada se dirige especifica y principalmente a dotar a los interesados de los conocimientos, habilidades y actitudes propias de la atención a la salud».

La regulación de las profesiones sanitarias implica una formación con perfiles específicos, que respondan a las necesidades del sistema sanitario y a los avances científico-técnicos. Esta delimitación no tiene porqué colisionar con el fomento del trabajo interdisciplinar, sino que lo potencia. En este sentido, la LOPS en su Exposición de motivos, apdo.II plantea "la cuestión de los ámbitos competenciales de las profesiones sanitarias manteniendo la voluntad de reconocer simultáneamente los crecientes espacios competenciales compartidos interprofesionalmente y los muy relevantes espacios específicos de cada profesión. Por ello en esta ley no se ha pretendido determinar las competencias de unas y otras profesiones de una forma cerrada y concreta, sino que establece las bases para que se produzcan estos pactos entre profesiones, y que las praxis cotidianas de los profesionales en organizaciones crecientemente multidisciplinares evolucionen de forma no conflictiva, sino cooperativa y transparente.»

La regulación es apropiada pues no tendría sentido que desde una ley que concier- 
ne a las profesiones sanitarias se pretendiera regular la estructura general de la formación y el desarrollo profesional de otros ámbitos y profesiones cuya actividad desborda el específicamente sanitario. Es además, conveniente para poder planificar adecuadamente las necesidades y disponibilidades de profesionales sanitarios del sistema de salud.

El desarrollo de actividades sanitarias exige ya una especialización y control de calidad desde el inicio mismo de la formación, por cuanto lo que está en juego es la intervención activa en la enfermedad, tanto en tratamiento como prevención. Es de destacar, en ese sentido, que el título de licenciado en medicina no habilita para el ejercicio en el sistema nacional de salud, sino que ha de completarse con una formación especializada

Así pues, el objetivo principal de la formación de los profesionales sanitarios es proporcionar una atención sanitaria de la máxima calidad y contribuir a mejorar el estado de salud. Para lograr este objetivo, los profesionales en formación deben ser progresivamente capaces de:

- Dar respuesta a las necesidades de los sistemas sanitarios y de la sociedad,

- Afrontar la continua irrupción de nuevos conocimientos y habilidades, así como los cambios que conllevan

- Asegurar una buena formación en las nuevas tecnologías de la información y comunicación, y en habilidades para el desarrollo del trabajo multidisciplinar.

- Fomentar la autocrítica, la curiosidad y la capacidad de aprender a lo largo de toda la vida profesional

- Ser capaces de desarrollar su actividad en un contexto interdisciplinar

- Desarrollar estilos de comunicación adecuados con los enfermos y su entorno, potenciando la colaboración activa de éstos en el manejo de sus enfermedades y la mejora de su salud

- Asumir cada vez mayores cotas de responsabilidad en su actividad clínica

Los cambios habidos en la atención sanitaria y las nuevas exigencias suscitan también la necesidad de cambios tanto en la estructura como en el proceso de la formación y educación sanitaria, de forma que aborden de forma concreta las demandas sanitarias de la poblacion y las insuficiencias que se manifiestan en la atención sanitaria, lo que presupone el mantener contacto apropiado con los pacientes y adquirir el conocimiento clínico y las habilidades para asumir la responsabilidad clínica.

Las consideraciones y la argumentación expuestas justifican una regulación y la delimitación de un ámbito específico de las profesiones sanitarias, que concierne a su formación y sus funciones, diferenciadas de las actividades que otras profesiones realizan en el campo de la salud y de la atención sanitaria.

\section{DIFERENCIAS ENTRE EL EJERCICIO DE PROFESIONES SANITARIAS \\ Y LA ACTIVIDAD DE OTRAS PROFESIONES EN EL ÁMBITO SANITARIO}

Para que una actividad profesional sea considerada como sanitaria, en el sentido a que se refiere el término sanitario en la LOPS, el objetivo principal y prioritario de tal actividad es el de restaurar hasta donde sea posible la salud perdida o alterada de las personas. Ello conlleva intervenir activamente en los procesos de salud / enfermedad, mediante procedimientos adecuados de evaluación, diagnóstico, tratamiento, y rehabilitación de la salud perdida, así como desarrollar actuaciones encaminadas a la prevención de enfermedades y, consecuentemente, la promoción de la salud.

Pero es una evidencia que en el campo de la atención a la salud intervienen, por las razones ya apuntadas de la progresiva complejidad y multidisciplinariedad, ejerciendo sus actividades otros profesionales sin que puedan definirse como profesiones sanitarias. Pues es evidente que aunque estas actividades son muy importantes para el avance y la mejora de la calidad de la atención sanitaria, desde el punto de vista de la regulación profesional tienen una estatus diferenciado. Así, es posible la presencia de un psicólogo en el 
SNS como gestor de RRHH, -recursos humanos- sin que ello le convierta o configure en un profesional sanitario.

En el conjunto de actividades profesionales de los psicólogos, la única inequívocamente sanitaria en el sentido apuntado, tanto en lo que se refiere a los contenidos de su formación ${ }^{7}$ como a su ejercicio es la que desempeñan los psicólogos clínicos.

En consecuencia, tanto desde el punto de vista del campo de conocimientos como desde el operativo y práctico se debe diferenciar entre el ejercicio de las profesiones sanitarias y el desarrollo de actividades en el campo sanitario de otras disciplinas y profesiones.

\section{LA CREACIÓN DE LA ESPECIALIDAD DE PSICOLOGÍA CLINICA Y LA REGULACIÓN DE SU SISTEMA DE FORMACIÓN COMO GARANTIA DE UN ALTO NIVEL PROFESIONAL}

La creación de la especialidad de Psicología clínica y su regulación ha supuesto un importante avance en nuestro país para asegurar una formación especializada de calidad y promover la incorporación del psicólogo clínico al sistema sanitario, como se viene haciendo desde hace años en los países de nuestro entorno y como se preconiza ya en el Informe de la Comisión Ministerial para al reforma psiquiátrica el año 1985.

El procedimiento adoptado de carácter teórico-práctico se ajusta al sistema vigente en nuestro país para la formación de especialistas sanitarios: el modelo de Interno- Residente en servicios sanitarios, acreditados a tal fin por el SNS. Este sistema, sin perjuicio de su mejora y desarrollo, se ha mostrado válido y ha adquirido un prestigio y reconocimiento que no deben ser devaluados ni menoscabados por otros procedimientos alternativos o complementarios. En definitiva, este procedimiento, que se reguló mediante el decreto de creación de la especialidad ( $R D$ 2490/1998) es

\footnotetext{
' Ver programa vigente de Formación de Especialistas en Psicología Clínica y el nuevo Programa aprobado por la Comisión Nacional de la Especialidad - pendiente de aprobación por la Administración sanitaria y educativa-.
}

una garantía de capacitación y de atención especializada al ciudadano que no puede eludirse ni ponerse en cuestión.

Ciertamente, en la actualidad el número de plazas que se convocan cada año para realizar la especialidad es insuficiente $y$ debería experimentar un incremento progresivo adaptado a las demandas que se estimen en el sistema sanitario y a la disponibilidad de recursos humanos para asegurar una formación de calidad en los servicios que, como Unidades Docentes Acreditadas, realizan la formación de especialistas en Psicología Clínica, formación que ha de cumplir los requisitos establecidos.

Otro aspecto importante es la conveniencia de revisar e introducir posibles cambios en la formación especializada -nuevo programa y aumento a cuatro años de su duración-y desarrollar las áreas de capacitación específica (ACEs) que se contemplan en la propia LOPS y que los cambios y nuevas necesidades del SNS requieren.

Las exigencias y cualificación profesional en el ámbito sanitario han crecido y se han diversificado enormemente en todo el mundo en las últimas décadas. Así que debe aplicarse el nuevo programa ya elaborado por la Comisión Nacional de la especialidad y, eventualmente, potenciar áreas de capacitación especifica, y en su caso nuevas especialidades, en algunas materias que en el ámbito internacional, y en el europeo en particular, han demostrado un gran potencial de crecimiento y una alta demanda social, tales como la Psicología Clínica de Niños y Adolescentes, la Psiconeurología Clínica, la Psicologia de la Salud y la Psicoterapia, por mencionar algunas de las más importantes y demandadas

Ante la estructuración de los estudios de Psicología en el ámbito europeo, desde el punto de vista académico puede contemplarse la posibilidad de crear a través de las nuevas titulaciones de postgrado (Real Decreto 56/2005) una titulación de Master en Ciencias de la Salud ${ }^{8}$ que completase la formación

\footnotetext{
${ }^{8}$ Los RD 55/2005 y 56/2005 establecen entre otras cosas que no podrán las titulaciones de Master incorporar en su denominaciones las de las especialidades sanitarias.
} 
de pre-grado y diera a sus titulados formación específica en ese ámbito.

El reconocimiento de capacitación diferencial que otorga el título de especialista en Psicología Clínica vía sistema PIR, respecto al que resultará del futuro grado en Psicología y master en CC de la Salud, debería contemplarse como el nivel de titulación requerida para acceder al concurso de plazas de formación de especialistas. Este último aspecto cobra especial relevancia si tenemos en cuenta que las nuevas titulaciones de grado en Psicología tendrán una carga lectiva menor, según lo establece el Convenio de Bolonia. Al finalizar la formación de grado y un postgrado de master en Ciencias de la Salud, los titulados se hallarían en una situación similar a la de otros estudios de Ciencias de la Salud antes de iniciar la formación especializada.

\section{SOBRE LA NECESIDAD Y DEMANDA DE PSICÓLOGOS CLÍNICOS EN EL SISTEMA NACIONAL SANITARIO}

El Sistema Sanitario Español ha incorporado y reconocido a los psicólogos clínicos como especialistas muy recientemente (en 1998, aunque sólo en el 2002 se inicia la operativización del proceso). La necesidad y demanda de psicólogos especialistas en psicología clínica en el SNS supera hoy por hoy la presencia de estos profesionales cualificados, porque hay unas dotaciones que son insuficientes y muy desiguales. La media de psicólogos en los servicios de salud mental es 4,28 por 100.000 habitantes, cuando algunas estimaciones recomiendan ratios de $11^{9}$. El número de psicólogos clínicos en el sistema sanitario no ha experimentado prácticamente incrementos en la última década y esto tiene una repercusión negativa en la formación de los equipos multidisciplinares y en el desarrollo de un práctica integral que comprenda los aspectos bio-psico-sociales de la persona.

Se considera urgente proceder a la consolidación y clasificación de estos profesiona-

\footnotetext{
${ }^{9}$ Datos del observatorio de salud mental de la AEN. Diciembre 2003.
}

les 0 , que en muchos casos desempeñan sus funciones desde hace más 20 años, como facultativos especialistas, así como ampliar las plazas de facultativos especialistas en psicología clínica dentro del SNS, lo que permitirá incrementar a su vez las plazas para la formación de futuros especialistas mediante el sistema de residencia (PIR).

No obstante, hay que tener en cuenta también que cerca de 10.500 psicólogos han solicitado ser reconocidos como especialistas en psicología clínica. De ellos, la Comisión Nacional de Psicología Clínica, ha revisado e informado hasta la fecha más de 3.000 , y todo ello en un plazo de 2 años escasos, lo que desde luego indica la gran implicación de las personas que forman esa Comisión y el excelente trabajo que vienen desarrollando. Además, la reciente publicación del nuevo RD $654 / 2005$ que amplía el plazo para poder solicitar la homologación, producirá un incremento importante en el número de solicitudes. Ante esta nueva demanda será necesario aplicar medidas que ayuden a la Comisión Nacional a agilizar el estudio de los expedientes y el reconocimiento, cuando proceda, de los correspondientes títulos de especialista creando así la posibilidad de mejorar las dotaciones y la disponibilidad de psicólogos especialistas en psicología clínica en el sistema sanitario.

\section{SÍNTESIS Y CONCLUSIONES}

La tradición científica y profesional de la psicología es mucho más amplia que la que se circunscribe al campo la salud. Se extiende desde el diseño de culturas, la ingeniería comportamental para los sistemas de producción, pasando por el estudio de la psicología de las concepciones del mundo, los estereotipos nacionales, las diferencias individuales en actitudes y aptitudes o competencias humanas, la promoción del bienestar social, promoción de instrucción eficiente en distintos campos que van desde la psicología del astronauta hasta la de las discapacidades humanas y la superdotación y genialidad, hasta cuestiones relacionadas con la prevención de trastornos mentales, promoción de la salud y tratamientos de casos individuales, 
psicología de la religión y un largo etcétera. Una restricción de todo el campo al mundo de la salud cercenaría en nuestro país radicalmente y de modo incomprensible muchos de los desarrollos de la psicología contemporánea.

Del análisis y estudio llevado a cabo para la realización de este informe extraemos las siguientes conclusiones:

1. El análisis del decreto que establece las directrices de los planes de estudio de la licenciatura de psicología, el volumen y la distribución curricular de materias, los contenidos de troncalidad de carácter sanitario y relacionados con la salud 0 , la definición y organización del practicum y la comparación con el perfil de otras Licenciaturas inequívocamente sanitarias, permiten concluir que esa licenciatura no ha sido concebida ni estructurada como una formación principalmente sanitaria.

2. De lo expuesto hasta aquí podemos concluir que, desde el punto de vista de la formación curricular del título de licenciado en Psicología, no puede considerarse que los titulados hayan recibido una formación específica ajustada al ámbito de las profesiones sanitarias reguladas, ni tampoco que su actividad profesional se limite al ámbito sanitario y, por consiguiente, no es ajustado a razón ni apropiado considerar al licenciado en psicología como una profesión sanitaria regulada.

3. El estudio comparado de la formación de la licenciatura de psicología en los diferentes países de la UE, así como los procedimientos para el acceso al ejercicio profesional nos muestra que también en estos países la licenciatura de psicología está considerada y estructurada con un carácter generalista y polivalente. Las asociaciones científicas y profesionales europeas de la Psicología formulan de forma explícita la necesidad de realizar estudios post-licenciatura, con un formato específico y de una duración regulada, para trabajar como psicólogo en contextos sanitarios.
4. El modelo integral de atención a la salud defendido por la OMS y definido como modelo bio-psico-social, del que participa la psicología, no significa que toda la actividad que se pueda desarrollar en los ámbitos biológicos, psicológicos o sociales, esté directa y exclusivamente asociada a la mejora y protección de la salud. Lo que expresa ese modelo, en lo que a la atención y cuidado de la salud se refiere, es que ésta debe ser atendida y contemplada en esas tres vertientes y de manera coordinada, para lo cual es necesaria la interdisciplinariedad.

Considerar sanitaria una actividad profesional de mejora del rendimiento, de adaptación escolar o educativa, un estudio de mercado, o una selección de personal, implica no solo un desconocimiento del sistema sanitario y sus exigencias, sino además una simplificación y reducción de la diversidad de roles profesionales de los psicólogos y de la concepción de la atención sanitaria a los ciudadanos.

Hay actividades de orden económico, social, de saneamiento o urbanístico que sin duda repercuten en la salud, pero no por ello es fundamentado considerar a los profesionales de esos campos como profesiones sanitarias. Siendo la salud un elemento importante en la vida del ser humano, esta salud está condicionada por muchos factores y posee muchas vertientes - por ejemplo, la economía, la sociología, la política-, y no es razonable considerarlas por esta influencia como ciencias de la salud.

5. La complejidad actual de las intervenciones que se realizan en el campo sanitario hace necesario que concurran en ellas disciplinas y profesiones diferentes de las estrictamente sanitarias y que se pueda diferenciar entre actividades sanitarias ejercidas por profesionales sanitarios y otras que se aplican en este ámbito por profesiones distintas, no sanitarias.

6. En España se dispone de un sistema de formación para la obtención del 
título de especialista en Psicología clínica, el modelo interno-residente, que tiene un reconocido prestigio y garantiza la competencia de estos profesionales para desarrollar su actividad en el sistema sanitario y contribuir a prestar una atención de calidad. Ninguna iniciativa debe poner este sistema en riesgo; por el contrario deben estudiarse las mejoras posibles, revisando y actualizando el programa de formación y su duración, e iniciar el desarrollo de las áreas de capacitación específica para dar respuesta, en la medida de lo posible, a la complejidad de las intervenciones psicológicas que se requieren en el sistema sanitario.

7. Es preciso adoptar medidas que agilicen la tramitación y faciliten a la Comisión Nacional de la Especialidad el estudio y resolución de los expedientes que están en proceso de homologación, en aplicación de los procedimientos transitorios de acceso al título que se han regulado en el real decreto 2490/1998.

Se han presentado más de $\mathbf{1 0 . 0 0 0}$ solicitudes, de las que se han resuelto ya más de 3000, y ese número se va a ver incrementando con los cambios que introduce el RD 654/2005; además es necesario que el número de plazas de PIR experimente un aumento progresivo. En consecuencia, con la aplicación de todas estas medidas, la disponibilidad de psicólogos especialistas en psicología clínica mejorará y alcanzará un número razonable.

8. Las necesidades percibidas en el SNS y las ratios actuales de estos profesionales en el propio sistema, hacen necesario incrementar las dotaciones de psicólogos clínicos y consolidar su situación como facultativos especialistas. De ese modo se podrá hacer frente tanto a dichas necesidades, como a la exigencia de las dotaciones apropiadas de tales profesionales en las Unidades Docentes Acreditadas para la formación de especialistas en Psicología Clínica.
Es preciso, también, aprobar y poner en marcha el nuevo programa de la especialidad, estudiar su posible ampliación a cuatro años, y desarrollar las Áreas de Capacitación Específica (ACEs), en aquellos ámbitos en los que la demanda de formación y capacitación es más evidente y está científicamente más consolidada.

9. El R.D 1277 de 2003 que establece las bases generales sobre autorización de centros, servicios y establecimientos sanitarios, no es claro en su definición de los servicios y unidades, ha dado lugar a interpretaciones y aplicaciones impropias, y maneja conceptos extemporáneos, por lo que debe ser revisado para su modificación. Sobre la conveniencia de esta revisión hay acuerdo entre diversas asociaciones y organizaciones de profesionales de la Psicología y hay también un pronunciamiento en ese sentido de la Comisión Técnica de Formación especializada en Ciencias de la salud

10. Ante la nueva regulación de los estudios del grado y postgrado que deben producirse en el futuro inmediato para adecuar los actuales estudios de psicología a las normativas europea y española (R.D.56/2005), deberá estudiarse en su momento, si, en razón de los contenidos del postgrado, procede realizar modificaciones en la catalogación de esos estudios y reconocerles un carácter sanitario. También se deberán definir entonces, con claridad, los requisitos para el acceso a la formación de especialistas en psicología clínica, que podrán incluir la formación de grado más la realización de un Master específico en ciencias de la salud.

\section{RECOMENDACIONES}

1. En las circunstancias actuales no es razonable considerar a la licenciatura en psicología, que tiene un carácter polivalente y generalista, como una profesión sanitaria regulada y, en consecuencia, no proce- 
de realizar ningún cambio en la LOPS a ese efecto.

2. Es necesario modificar varios contenidos del Real decreto 1277/ 2003,de 10 de Octubre, por el que se establecen las bases generales sobre autorización de centros, servicios y establecimientos sanitarios, corrigiendo adecuadamente materias relacionadas con las definiciones de centros y unidades, así como los requisitos y el ámbito de aplicación

3. Se debe fomentar el trabajo multidisciplinar en el ámbito del Sistema Nacional de Salud, mejorando las dotaciones, y la formación y habilidades para desarrollar esa actividad multidisciplinar.

4. Se debe potenciar y seguir desarrollando la formación de especialista en psicología clínica, a través el sistema PIR, evitando que prosperen otras alternativas que contribuyan a su erosión o devaluación.

5. Se debe aplicar el nuevo programa de formación elaborado y aprobado por la Comisión de la Especialidad y desarrollar los procedimientos necesarios para la formación y capacitación en áreas específicas ACEs - que respondan a las necesidades del sistema sanitario.

6. Se deben adoptar medidas para agilizar la tramitación y facilitar a la Comisión Nacional de la especialidad la resolución de los expedientes presentados para la homologación del título de especialista en psicología clínica, de acuerdo con las disposiciones transitorias del real decreto 2490/1998, que se verán incrementados con la ampliación del periodo computable que establece el reciente R.D. 654/ 2005

7. Se debe fomentar la mejora de las dotaciones de psicólogos clínicos en el Sistema Nacional de Salud que en la actualidad se mantienen aún en ratios bajas. Así mismo, es necesario que, en todas las CCAA, se proceda a la reclasificación de los psicólogos clínicos como facultativos especialistas

8. En función del aumento del número de centros acreditados para la formación y de las necesidades del sistema de salud, se debe incrementar progresivamente las plazas de PIR y mejorar la ratios de plazas/aspirantes.

\section{ANEXO I \\ Regulación de los estudios de Psicología en diversos países europeos}

- Alemania: Para obtener el Diploma en Psicología, es necesario haber cursado 10 semestres (5 años), siendo uno de ellos de prácticas. Los 4 semestres primeros (Prediploma) no cualifican para el ejercicio profesional; Los 6 siguientes, terminan con un examen que incluye Tesis de investigación. Una vez obtenido el Diploma, se puede acceder al título de Magíster y al Examen de Estado, que implican cursos adicionales para ejercer profesionalmente de manera especializada. No obstante, solo están regulados los tiempos mínimos necesarios para la especialización profesional en Psicología Clínica-Psicoterapia (3 años) o en Psicología del trabajo y las organizaciones (2 años).

- Bélgica: 5 años para acceder al título. Habilita para el ejercicio profesional en todos los campos de la psicología excepto en Psicología Clinica, para los que se requieren 3 años adicionales con prácticas supervisadas.

- Dinamarca: Se requieren 8 años de estudios. Sigue el esquema $3+2+3$ : el primer ciclo es generalista, no capacitando para el ejercicio profesional. El segundo ciclo, equivalente al Master, capacita para el ejercicio profesional supervisado (no independiente) en el área del Master que se haya cursado. El tercer nivel ( 3 años) es necesario para el ejercicio profesional independiente en alguna de las áreas de la Psicología (Psicoterapia, Clínica, Infantil, Escolar, Neuropsicología, Gerontopsicologia, Salud).

- Finlandia: Para acceder al título se requieren entre 7 y 9 años. Sigue el modelo Bachelor ( 3 años, no capacita para ejercicio profesional), + Master (2 años, capacita para ejercicio profesional en el master elegido, pero sin categoría de especialista), + 2 o 4 años (Grado superior de Licenciado, necesario para práctica independiente y especializada en una de estas áreas: Educativa y del desarrollo, Salud, Psicoterapia, Neuropsicología, y Ps. Del Trabajo).

- Francia: Para acceder al Título, se requieren 6 años en 4 ciclos diferenciados. Pri- 
mero: 2 años (diplomatura, generalista, no especializada, no práctica profesional); Segundo: 1 año (estudios específicos en un área de la psicología, no capacita para ejercicio profesional independiente); Tercero, Formación especializada (maestría, 2 años, necesaria para trabajo profesional independiente en el campo elegido); Cuarto (1 año, práctica profesional supervisada). Algunas especialidades (Clínica, Educativa) tienen requisitos específicos de formación adicionales, que configuran un quinto ciclo

- Grecia: Para acceder al título se requieren entre 6 y 7 años, en dos ciclos: el primero de 4 años que no capacita para ejercicio profesional en ningún ámbito, pese a incluir en el cuarto año prácticas y tesis de investigación; segundo ciclo, de 2 o 3 años, para cursar una especialidad que necesariamente debe comportar una fuerte carga de prácticas supervisadas. Especialidades vigentes: Clínica, Escolar, Organizaciones, Desarrollo, Ps. Economica.

- Irlanda: Entre 5 y 7 años (según Universidades) en dos ciclos: Grado (3 o 4 años, formación básica y generalista) y Master (2-3 años, según especialidad escogida y universidad). Hay postgrados en Clínica, Consejo Psicológico, Educativa, Forense, Salud, Organizacional. Para obtener el título e inscribirse como tal en el Colegio profesional (Psychological Society of Ireland), esta organización exige realizar un examen que si es aprobado da lugar a la obtención del Diploma en Psicología Profesional en una de dos especialidades posibles: Clínica y Educativa.

- Italia: Para obtener el título son necesarios 6 años (el último de prácticas supervisadas). Posteriormente, se puede ejercer en cualquier ámbito profesional, excepto en Psicoterapia, para el que se exigían 4 años más posteriores a la licenciatura ("Laurea»).

- Portugal: En general, 5 años para el título, organizados en tres ciclos: 3 años de contenidos generalistas, 1 año de iniciación en algún ámbito profesional, 1 año de prácticas. Los títulos que se obtienen son generalistas. Hay un título diferenciado para Psicología Clínica, que se imparte en el
Instituto Superior de Ciencias de la Salud (CESPU) y que consta de 5 años.

- Reino Unido: Para la obtención del título se requiere cursar un mínimo de 6 años, distribuidos en Bachelor ( 3 o 4 años) + Postgrado (3 o más años) para las diferentes especialidades. El Bachelor es generalista y no capacita para el ejercicio profesional. El Postgrado da opción a obtener el Chartered Psychologist (análogo a la Licenciatura española), que permite el acceso al ejercicio profesional, en el campo o área específica que se haya cursado, y que se acredita como en Irlanda mediante un examen de la British Psychological Society. El título de Chartered se suele adjetivar en función de la especialidad cursada: Chartered Clinical Psychologist, hatered Educational Psychologist, etc. El nivel máximo de especialización se adquiere posteriormente a este título, mediante estudios adicionales. En el caso de la Psicología Clínica, se requieren 3 anos de dedicación exclusiva, y da acceso a la obtención del título de Doctor en Psicología Clínica.

- Suecia: Para el acceso al título de Licenciado se requieren 5 años +1 de práctica supervisada. El título capacita para ejercer en cualquier ámbito de la psicología, excepto en el de Psicología Clínica (Psicoterapia), para el que se requiere una capacitación específica de al menos 2 años.

\section{DOCUMENTACIÓN Y BIBLIOGRAFÍA}

\section{Documentos del Colegio Oficial de Psicólogos}

1998. Perfiles profesionales del psicólogo. Papeles del Psicólogo www.cop.es/index/html

1999. PIR. Formación de especialistas en Psicología Clínica. Papeles del Psicólogo, $1999, n^{Q} 43$.

2003. A fondo: Psicología Clínica. Papeles del Psicólogo, 2003, 24 (85).

2003. Dictamen Jurídico: La inclusión de psicología en el art.2.2 a ) del proyecto de Ley de Ordenación de Profesiones Sanitarias, al objeto de ser amparado todo el colecti- 
vo sin excepción.. 3 de octubre de 2003 Colegio Oficial de Psicólogos de Castilla y León. 2004.

2004. Comunicado de la Junta de Gobierno del Colegio Oficial de Psicólogos ante la normativa que regula el ejercicio de la Psicología en España. Suplemento informativo de Papeles del Psicólogo, $\mathrm{n}^{\mathrm{9}} 86$.

2004. Los psicólogos somos profesionales sanitarios. INFOCOP, $\mathrm{N}^{2}$ Extraordinario. 2004. LOPS: Cronología de un conflicto. INFOCOP, $\mathrm{n}^{\mathrm{2}} 21,26-39$.

2005. La Psicologia es una profesión sanitaria. INFOCOP, $\mathrm{n}^{9} 22$.

2005. INFOCOP (Marzo). № extraordinario sobre la LOPS.

2005. Santolaya, F. Editorial. INFOCOP, $\mathrm{n}^{\circ} 23$, p. 2 .

2005. Santolaya, F. El COP a lado de la Psicología y de la especialidad de Psicología Clínica: ¿por qué no trabajamos todos juntos?. INFOCOP, $\mathrm{n}^{\mathrm{2}} 23,114-117$ (Cartas al Director).

2005 (Abril). Informe dirigido al Subsecretario de Sanidad del Ministerio de Sanidad y Consumo, de la Junta de Gobierno del Colegio Oficial de Psicólogos.

\section{Documentos de la Conferencia de Decanos de Psicología}

2004. Libro Blanco sobre la Titulación. ANECA, 2004.

2005. Declaración de la Conferencia de Decanos de Psicología, reunida en Murcia, 28-29 de Abril, 2005.

Documentos de Asociaciones:

AEN, AEPCP, ANPIR

Asociación Española de Psicología Clínica y Psicopatología (AEPCP). Informe de la AEPCP sobre el debate en tomo a la Ley de Cohesión y Calidad del SNS y la LOPS. Revista de Psicopatología y Psicología Clínica, 2004, 9 (1), 71-78.

AEN, AEPCP, ANPIR, COPAO, COPG. Declaración por el desarrollo de la psicología clínica en España. Revista de Psicopatología y Psicología Clínica, 2004, 9 (3), 255-258.
AEN (Asociación Española de Neuropsiquiatría). Declaración sobre la identidad sanitaria de la psicología clínica y el carácter polivalente de la licenciatura de psicología. Madrid 28.1, 2005.

ANPIR: Comunicado sobre la LOPS y la licenciatura de psicología. Madrid 22, diciembre, 2004.

Espino A, Olabarria B. La formación de los profesionales de la salud mental en España. $A E N$. Madrid 2003.

González-Blanch C, Jiménez A, Palacios AJ. La Psicología Clínica en los tiempos de la LOPS. INFOCOP, $\mathrm{n}^{9} 23,111-116$.

Olabarría, B. (2004). Avatares de la Psicología Clínica, especialidad sanitaria de la Psicología. Revista de Psicopatología y Psicología Clínica, 2004, 9 (2), 163-174.

\section{Otros Documentos}

Una propuesta marco para la educación y la formación del psicólogo en Europa. Documento elaborado por el Proyecto EuroPsyT www.eeuropsy.org

Resolución adoptada por el Defensor del Pueblo con motivo de la interposición de recurso de inconstitucionalidad contra la ley 44/2003, de 21 de Noviembre, de ordenación de las profesiones sanitarias. Madrid 20 Febrero,2004.

Peiró J.M. La enseñanza de la psicología en Europa Un proyecto de titulación europea. Papeles del psicólogo,86,2003.

McFall, R. Manifesto for a science of Clinical Psychology. The Clinical Psychologist, 1991, 44 (6), 75-88.

Informe de la Comisión Ministerial para la reforma Psiquiátrica. MSC. Servicio de publicaciones. Abril 1985.

Comisión Nacional de Psicología Clínica. Programa para la formación de especialistas en psicología clínica. Marzo 2003

\section{Normativa oficial}

BOE 286, de 30/11/1999. Ministerio de educación y Cultura. Orden de noviembre de 1999 por la que se determinan los estudios conducentes a la obtención de títulos uni- 
versitarios oficiales que se relacionan con cada de una de las vías de acceso a dichos estudios

BOE 288, de 2/12/1998. Real Decreto 2490/1998, de 20 de Noviembre, por el que se crea y regula el título oficial de psicólogo especialista en psicología clínica

BOE 119, de 18/5/2002. Orden Pre/1107/ 2002 de 10 de Mayo, por la que se regulan las vías transitorias de acceso al título de Psicólogo especialista en psicología clínica.
BOE 254, de 23/10/2003. Real Decreto 1277/ 2003 de 10 de Octubre por el que se establecen las Bases generales sobre autorización de centros, servicios y establecimientos sanitarios

BOE 280, de 22/11/2003. Ley 44/ 2003 de 21 de noviembre, de Ordenación de las Profesiones Sanitarias

BOE 142, de 15 de Junio. Real Decreto 654/ 2005, de 6 de Junio, por el que se modifican las disposiciones transitorias del Real Decreto 2490/ 1998 